\title{
Graph Theory Based Beam Scheduling for Inter-cell Interference Avoidance in MmWave Cellular Networks
}

\author{
Ziyuan Sha, Zhaocheng Wang, Senior Member, IEEE, Sheng Chen, Fellow, IEEE, and Lajos Hanzo, Fellow, IEEE
}

\begin{abstract}
The application of high directional beam in millimeter wave leads to a new downlink inter-cell interference (ICI) characteristic that the ICI is high only if the beam of a neighboring cell points towards a user in local cell. This characteristic indicates that the ICI can be avoided if the beams in the network are scheduled coordinately. In this paper, we investigate the time-domain beam scheduling problem for downlink ICI avoidance by modeling the entire network as a graph, where the number of time slots occupied by each beam is considered as a constraint to guarantee service quality. The beams in each cell are classified according to the neighboring cells they may interfere with. If two adjacent cells simultaneously use the beam which may interfere with each other, beam collision occurs, leading to strong ICI. Based on graph theory, we propose a least beam collision (LBC) algorithm to minimize the number of beam collisions, and we prove that this LBC algorithm is capable of acquiring the global minimum beam collision solution. Our simulation results verify that the strong ICI between two neighboring cells can be efficiently eliminated, which benefits the transmission reliability and the network's sum rate.
\end{abstract}

Index Terms-Millimeter wave, beamforming, inter-cell interference, beam scheduling, beam collision, graph theory

\section{INTRODUCTION}

Millimeter wave (mmWave) band is currently considered as a promising frequency band for future communication systems due to its abundant bandwidth. For mmWave systems, high directional power gain provided by large antenna array is necessary to mitigate the severe path loss in the mmWave band [1]-[4]. However, because of the hardware constraint, the fulldigital multi-input multi-output (MIMO) transceiver architecture is no longer achievable [2]. Instead, analog beamforming technique based on phase shifter is typically utilized to meet the hardware constraint in mmWave communication [5]-[8].

In analog beamforming, a radio frequency (RF) chain is connected to an antenna array through phase shifters, and the

Z. Sha and Z. Wang are with Beijing National Research Center for Information Science and Technology, Department of Electronic Engineering, Tsinghua University, Beijing 100084, China. Z. Wang is also with Division of Information Science and Technology, Graduate School at Shenzhen, Tsinghua University, Shenzhen 518055, China (E-mails: shazy17@mails.tsinghua.edu.cn, zcwang@tsinghua.edu.cn).

S. Chen and L. Hanzo are with School of Electronics and Computer Science, University of Southampton, Southampton SO17 1BJ, UK. S. Chen is also with King Abdulaziz University, Jeddah 21589, Saudi Arabia (E-mails: sqc@ecs.soton.ac.uk, lh@ecs.soton.ac.uk).

This work was supported in part by National Natural Science Foundation of China (Grant No. 61571267), in part by Shenzhen Fundamental Research Project (JCYJ20170817160741066), in part by Shenzhen Wireless over VLC Technology Engineering Lab Promotion, and in part by the Royal Society's Global Research Challenges Fund.

L. Hanzo would like to acknowledge the financial support of the Engineering and Physical Sciences Research Council projects EP/Noo4558/1, EP/PO34284/1, COALESCE as well as of the European Research Council's Advanced Fellow Grant QuantCom. weights on phase shifters form a steering vector which steers the direction of beam. To reduce the complexity of acquiring steering vector, codebook based analog beamforming is usually adopted [5], [6]. The steering vector is selected from a predefined codebook which includes multiple steering vectors corresponding to different directions of beam. The transmitter and receiver can jointly perform a beam training procedure to determine which beam is selected from the codebook, and such a joint transmit and receive beam training plays a key role in $5 \mathrm{G}$ cellular network [6].

Due to the application of high directional beam, the downlink inter-cell interference (ICI) in mmWave network mainly depends on the direction of beam used by neighboring cell, and this is different from lower frequency systems where the radiation pattern of antenna tends to be omni-directional. Unless the beam from a neighboring cell points towards a local user, low ICI can be achieved. According to [9]-[12], the performance of mmWave network is shown to be noiselimited rather than interference-limited when the cell density is low. However, for dense network, the ICI will limit the network performance because of the increased ICI power.

Typically, signal processing techniques in physical (PHY) layer are adopted as efficient means for combating ICI [14][16], where the ICI is usually eliminated via the null space projection of ICI signal. However, in this paper, we focus on the scheduling methods for mitigating ICI in medium access control (MAC) layer. Compared with PHY based methods, MAC based methods attempt to avoid ICI instead of cancelling ICI. Hence, unlike PHY based methods whose performance critically depends on the accuracy of the channel state information (CSI), the precision of CSI required by MAC based methods can be coarser.

\section{A. Related Works}

Many state-of-the-art researches on mitigating the ICI of mmWave network are based on network sum rate optimization [17]-[22]. For example, the link arrangement problems between base stations (BSs) and users are investigated in [17], [19], [21], under different scenarios. The coordinated frequency resource allocation problem is investigated in [18].

Obviously, the sum rate is an important network performance metric, but optimizing sum rate causes two problems. Firstly, the global CSI between multiple BSs and multiple users over the whole network is required, and this induces frequent CSI exchanging within the network, which further imposes large transmission overhead as well as large latency. Secondly, optimizing the network's sum rate usually imposes 
high computational complexity and it is generally very challenging to find the global optimal solution.

Apart from sum rate optimization, the studies [23], [24] investigate muting protocol, where an interfering cell is muted if its beam may cause ICI to an user in neighboring cell. The muting protocol is similar as the ICI coordination (ICIC) mechanism in LTE [13], [25], which mutes a BS causing ICI or lower its transmitting power on specific time or frequency resources. Both the muting protocol and ICIC mechanism are efficient to avoid ICI with low complexity, but the network's throughput is sacrificed because of the muted resources.

Another issue is that the time-domain behavior of mmWave network is seldom considered in the state-of-the-art researches, while it is common for one cell to serve different users at different time slots, and different time-domain schedules of beams can significantly influence the ICI level in the network. [20] studies the time-domain beam scheduling problem ensuring fairness among different users, i.e. every user in a cell is assumed to occupy the same amount of time slots. However, this assumption is not universal enough because different users usually have different demands of service in practical systems. When we allow the service demands to be different, the scheduling problem has to be solved under diverse service demands which are arbitrary rather than fixed. Hence, we have to deal with diverse possibilities resulting from different service demands, which makes the scheduling problem more complex to solve. To the best of our knowledge, there has been no prior research on mmWave ICI considering the different service demands of users in time domain.

\section{B. Our Contributions}

In this paper, we develop a new time-domain beam scheduling scheme for avoiding the downlink ICI in mmWave networks. We define beam collision as the event that a pair of neighboring cells simultaneously use the beams which may interfere with each other. By avoiding beam collision, the occurrence of strong ICI between two neighboring cells can also be avoided. Therefore, our goal is to minimize the number of beam collisions in the network. In contrast to the muting protocol or the ICIC mechanism of LTE, our timedomain beam scheduling merely adjusts the time sequence of the beams, which would not sacrifice the throughput of the network.

Furthermore, in contrast to sum rate optimization, minimizing the number of beam collisions only has to consider the beams at the network side, hence the beam scheduling could be a purely network-side operation, which is easy to implement. Moreover, in our beam scheduling, the users do not have to measure the downlink CSI of multiple neighboring cells, which significantly reduces the pilot overhead in the network.

To obtain the beam scheduling with the minimum number of beam collision, we utilize graph theory to model the entire network and the beam collision events between neighboring cells. Specifically, each cell is represented by a node in a graph which represents the network. For two neighboring cells, the beams which may cause ICI between these two cells form an edge connecting the two nodes. If two adjacent cells simultaneously use the beams contained in the edge connecting them, one beam collision occurs and strong ICI might be encountered between the two cells. Furthermore, we allow the beams in a specific cell to occupy different numbers of time slots to serve the users, and the number of time slots occupied by each beam is considered as the service demand constraint in our scheduling problem. Then, we propose least beam collision (LBC) algorithm to solve our scheduling problem and prove that the LBC algorithm is capable of acquiring the global minimum number of beam collisions. Moreover, the minimum number of beam collisions has a simple mathematical expression, which reveals that beam collisions can be completely avoided in most cases, i.e., the minimum number of beam collisions is zero.

Our main contributions are summarized as follows.

- Based on graph theory, we propose a new framework to mitigate mmWave ICI via time-domain beam scheduling. The downlink CSI is not required in the beam scheduling after our graph model has been established.

- We allow the time-domain service demands of different beams to be different, and propose the LBC algorithm for solving the beam scheduling problem. The LBC algorithm is proved to be capable of acquiring the global minimum number of beam collisions.

- The expression of global minimum number of beam collisions indicates that this number would be zero in most cases, hence having the strong ICI between two neighboring cells completely eliminated.

- Our simulations verified that the strong ICI between two adjacent cells can be efficiently eliminated, and only the weak ICI impinged from remote cells may affect the users' signal-to-interference-plus-noise ratio (SINR). The transmission reliability is remarkably enhanced, and the network sum rate is improved as well.

The rest of this paper is organized as follow. Section II introduces our system model. The problem formulation and preliminaries are provided in Section III. Section IV proves the existence of the global minimum beam collision solution of our scheduling problem and proposes the LBC algorithm to acquire this solution. Simulation results are shown in Section V, and Section VI offers our conclusions.

\section{SYSTEM MODEL}

We assumed that the network consists of $K$ cells indexed from 1 to $K$, and the $k$ th cell has $m_{k}$ beams in its codebook indexed from 1 to $m_{k}$. We consider a time period consisting of $N$ time slots for beam scheduling, and each cell uses one beam at each time slot. Furthermore, the beam training procedure is assumed to be accomplished, hence the beam used for serving each user is considered determined.

\section{A. Beam Classification and Graph Construction}

The network is modeled as a graph $G$, where each cell is represented by a node. We denote the $k$ th cell as node $v_{k}$, and all the $K$ nodes form the node set $V=\left\{v_{1}, \cdots, v_{K}\right\}$. As illustrated in Fig. 1 (a), because of the geographic location difference, only a few beams with certain directions in a cell 


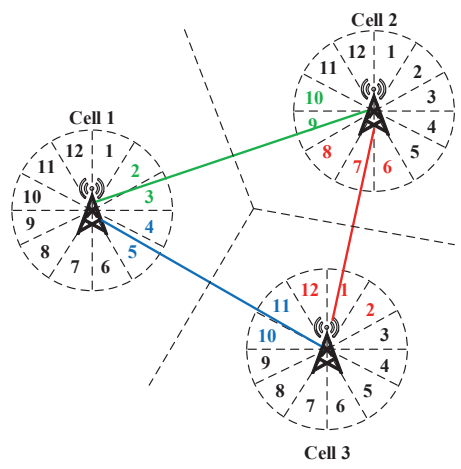

(a)

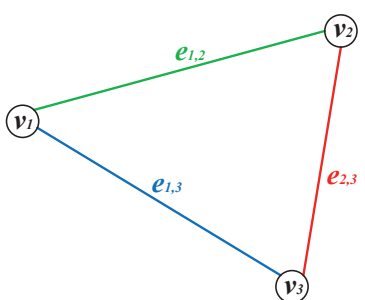

(b)
Fig. 1. Illustration of system model. The beams in different classes are marked with different colors: (a) network with $K=3$ and $m_{k}=12,1 \leq k \leq 3$, and (b) graph model of (a).

may cause ICI to a neighboring cell. Therefore, we can classify the beams in each cell according to the neighboring cell they may interfere with. Specifically, for cell $k$, the beams which may cause ICI to cell $k^{\prime}$ are classified into an interfering class denoted by the set $M_{k \rightarrow k^{\prime}}$, and the beams in cell $k$ which do not interfere with any neighboring cell are classified into an interference-free class denoted by the set $M_{k \rightarrow 0}$. For example, for cell 1 in Fig. 1 (a), since beams 2 and 3 may interfere with cell 2, we have $M_{1 \rightarrow 2}=\{2,3\}$. Similarly, we also have $M_{1 \rightarrow 3}=\{4,5\}$, and $M_{1 \rightarrow 0}=\{1,6,7,8,9,10,11,12\}$.

In our beam classification, we add a constraint that every beam belongs to only one class. Actually, few beams with particular directions might cause ICI to two neighboring cells. For example, in Fig. 1(a), beam 8 of cell 2 may interfere with both cell 1 and 3 . For a beam that may interfere with two neighboring cells, we only consider that it causes ICI to the neighboring cell closer to the direction of the beam, and its ICI to the other cell is treated as remote ICI and ignored. Thus, beam 8 in cell 2 only belongs to the class $M_{2 \rightarrow 3}$, since its ICI to cell 1 is smaller than its ICI to cell 3 . It can be seen that this constraint is actually a reasonable approximation.

As illustrated in Fig. 1(b), for two neighboring cells $k$ and $k^{\prime}$, the beams in $M_{k \rightarrow k^{\prime}}$ and $M_{k^{\prime} \rightarrow k}$ form an undirected edge $e_{k, k^{\prime}}$ connecting the two nodes $v_{k}$ and $v_{k^{\prime}}$. The set of all edges in $G$ is denoted as $E$, and we obtain $G=\{V, E\}$ as an undirected graph that represents the network.

In practical systems, the beam classification can be performed via user-aided interference measurement. For example, a cell-edge user served by beam 3 of cell 1 may measure a strong ICI from cell 2 and report it to cell 1 . Then, according to the time of the measured ICI, the network side can confirm the beam of cell 2 which caused the ICI, e.g. beam 9, and beam 9 would be added into class $M_{2 \rightarrow 1}$. Since the locations of BSs and the directions of the beams in codebook are both fixed, the structure of graph model can be long-term valid, so the overhead induced by the maintenance of the graph model would be low.

\section{B. Beam Service Demand and Class Demand}

Generally, the users in the network have different quality of service (QoS) requirements. To satisfy the QoS demands of different users, the upper layer of the network would determine a set of configurations of the communication resources, which includes the number of time slots allocated to each user. This then determines the numbers of time slots occupied by different beams. We denote the number of time slots demanded by the $m$ th beam in cell $k$ as $d_{k, m}$. Our objective is to perform time-domain beam scheduling to avoid ICI under arbitrary $d_{k, m}$ given by the upper layer.

Since the beams are classified into different classes, the class demand $\bar{d}_{k \rightarrow k^{\prime}}$ of class $M_{k \rightarrow k^{\prime}}$ is defined as the sum of the service demands of beams in $M_{k \rightarrow k^{\prime}}$, which can be expressed as

$$
\bar{d}_{k \rightarrow k^{\prime}}=\sum_{m \in M_{k \rightarrow k^{\prime}}} d_{k, m}, k^{\prime} \in \widetilde{V}_{k} \cup\{0\} .
$$

Here we denote the nodes' indices of all the adjacent nodes of node $k$ by the node index set $\widetilde{V}_{k}$, and all the beams in cell $k$ are divided into the sets $M_{k \rightarrow k^{\prime}}, k^{\prime} \in \widetilde{V}_{k} \cup\{0\}$. Because the total number of time slots is $N$ and every beam belongs to a unique class, the sum of class demands in each cell should also be $N$, which can be written as

$$
\sum_{k^{\prime} \in \widetilde{V}_{k} \cup\{0\}} \bar{d}_{k \rightarrow k^{\prime}}=N, k=1, \cdots, K .
$$

Furthermore, since the different beam classes in each cell are non-overlapped, the beams in the same class are treated identically in the following discussion. Therefore, we carry out the beam scheduling at class level rather than individual beam level.

\section{Beam Scheduling and Beam Collision}

The beam schedule scheme of $K$ cells over $N$ time slots is represented by the schedule matrix $\boldsymbol{B} \in \mathbb{N}^{K \times N}$. Since we perform the class level scheduling, the entry $b_{k, n}$ in the $k$ throw and $n$ th-column of $\boldsymbol{B}$ indicates that a beam in class $M_{k \rightarrow b_{k, n}}$ is used by cell $k$ at the $n$th time slot.

We define beam collision as the event that two adjacent cells simultaneously use the beams which may interfere with each other, i.e., if $\delta\left(b_{k, n}, k^{\prime}\right) \delta\left(b_{k^{\prime}, n}, k\right)=1$, then cell $k$ and cell $k^{\prime}$ cause a beam collision at time slot $n$, where the indicator function $\delta(x, y)$ is defined by

$$
\delta(x, y)= \begin{cases}1, & \text { if } x=y \\ 0, & \text { otherwise }\end{cases}
$$

Therefore, the total number of the beam collisions $n_{c}$ of a schedule scheme $\boldsymbol{B}$ can be written as

$$
n_{c}=\sum_{n=1}^{N} \sum_{e_{k, k^{\prime}} \in E}\left(\delta\left(b_{k, n}, k^{\prime}\right) \delta\left(b_{k^{\prime}, n}, k\right)\right) .
$$

Remark 1: The beam collision can be considered as a necessary condition of the strong ICI between two neighboring cells. Because of the uncertainties of user location and the non-ideality of transmission environment, e.g. blockage or reflection, a beam collision may or may not result in the occurrence of ICI. However, we can almost ensure that the strong ICI would not occur if the beam collision is avoided, which allows us to merely consider the beam at BS side and do not require the specific downlink channel state. Therefore, 
beam collision actually represents a possibility of strong ICI. If we manage to schedule the beams with out beam collision, we can eliminate the possibility of strong ICI between two neighboring cells.

\section{Beam Pattern}

The flat-top beam pattern [9], [11] is adopted at BS side. The directional power gain of beam is expressed as

$$
G(\phi)= \begin{cases}G_{\max }, & |\phi| \leq \frac{\phi_{b}}{2} \\ G_{\min }, & |\phi|>\frac{\phi_{b}}{2}\end{cases}
$$

where $\phi$ is the angle offset to the direction of a beam and $\phi_{b}$ is beamwidth, while $G_{\max }$ and $G_{\min }$ are the directional power gains when $\phi$ is inside and outside the beamwidth, respectively. At the user side, we consider the worst case that the omni-directional beam pattern is adopted, i.e. $G(\phi)=1$ for all $\phi$.

Remark 2: In practical systems, directional beam pattern might also be generated at user side. However, due to size and power limitation of hardware, the user beamwidth is typically very wide. Moreover, the user may intentionally use wide or omni-directional beam to simplify beam training and tracking procedure. Therefore, we consider the worst case that the user side uses omni-directional beam pattern [18], [20], [23], where the user is more susceptible to ICI. Besides, since our proposed beam scheduling is a purely network-side operation, which is independent of the beam pattern at user side, so our method is actually feasible for various user beam patterns.

\section{Problem Formulation AND Preliminaries}

\section{A. Problem Formulation}

To satisfy the service demand constraints, there must be $\bar{d}_{k \rightarrow k^{\prime}}$ entries equal to $k^{\prime}$ in the $k$ th row of $\boldsymbol{B}$, which can be expressed as

$$
\sum_{n=1}^{N} \delta\left(b_{k, n}, k^{\prime}\right)=\bar{d}_{k \rightarrow k^{\prime}}, k=1, \cdots, K, k^{\prime} \in \widetilde{V}_{k} \cup\{0\} .
$$

Therefore, given the graph $G=\{V, E\}$ with beam classification, by combining (2), (4) and (6), we can formulate our LBC scheduling problem as

$$
\begin{array}{ll}
\min _{B} & n_{c}=\sum_{n=1}^{N} \sum_{e_{k, k^{\prime}} \in E}\left(\delta\left(b_{k, n}, k^{\prime}\right) \delta\left(b_{k^{\prime}, n}, k\right)\right), \\
\text { s.t. } & \sum^{N} \delta\left(b_{k, n}, k^{\prime}\right)=\bar{d}_{k \rightarrow k^{\prime}}, \\
& k=1, \cdots, K, k^{\prime} \in \widetilde{V}_{k} \cup\{0\} .
\end{array}
$$

The class demands $\bar{d}_{k \rightarrow k^{\prime}}$ can be arbitrarily given as long as they satisfy (2) to ensure that the sum of class demands in each cell is $N$. We will refer to the scheduling problem (7) as a $(K, N)$ problem, where $K$ and $N$ are the number of cells and number of time slots, respectively.

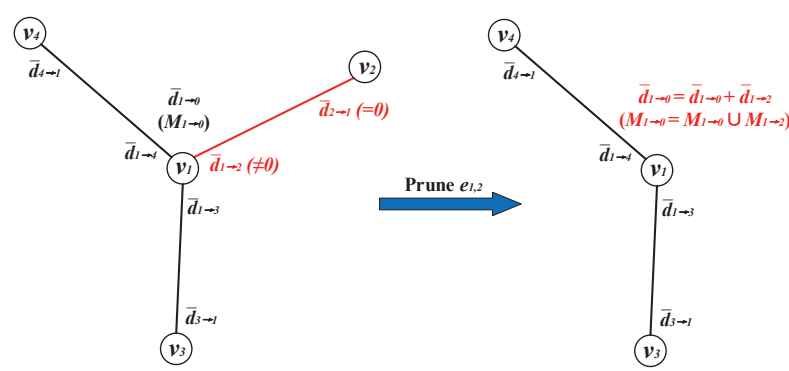

Fig. 2. An example of edge pruning. Since $\bar{d}_{1 \rightarrow 2} \neq 0$ and $\bar{d}_{2 \rightarrow 1}=0$, we can prune edge $e_{1,2}$ and add class $M_{1 \rightarrow 2}$ into the original interference-free class $M_{1 \rightarrow 0}$

\section{B. Edge Pruning for Interference-free Class Extension}

For two adjacent nodes $v_{k}, v_{k^{\prime}} \in G$, if $\bar{d}_{k \rightarrow k^{\prime}}=0$, the beams in $M_{k^{\prime} \rightarrow k}$ of cell $k^{\prime}$ never cause beam collision to cell $k$ because cell $k$ does not use the beams in $M_{k \rightarrow k^{\prime}}$. Therefore, we can include the beams in $M_{k^{\prime} \rightarrow k}$ in the interference-free class $M_{k^{\prime} \rightarrow 0}$ by pruning (removing) edge $e_{k, k^{\prime}}$. In this way, the original interference-free class $M_{k^{\prime} \rightarrow 0}$ is extended. An example of edge pruning for interference-free class extension is illustrated in Fig. 2. In this case, since $\bar{d}_{2 \rightarrow 1}=0$, we can prune edge $e_{1,2}$ and extend $M_{1 \rightarrow 0}$ by adding the beams in $M_{1 \rightarrow 2}$ into $M_{1 \rightarrow 0}$.

The procedure of edge pruning over the entire graph $G$ is summarized in Algorithm 1.

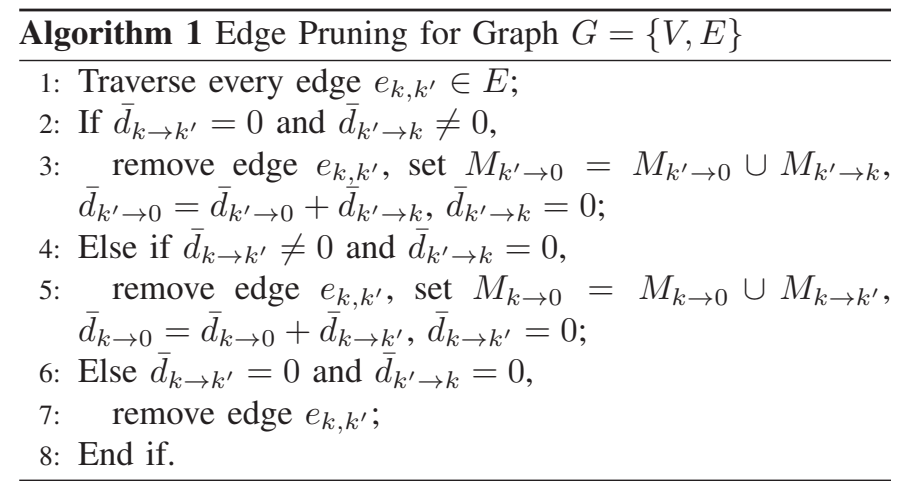

The following proposition for the graph after edge pruning is obviously holds.

Proposition 1. After edge pruning, for an arbitrary edge $e_{k, k^{\prime}} \in E$, we always have $\bar{d}_{k \rightarrow k^{\prime}} \neq 0$ and $\bar{d}_{k^{\prime} \rightarrow k} \neq 0$.

\section{Graph Theory Preliminaries}

We provide several fundamental results in graph theory, where the graph is assumed to be an undirected graph. For readers interested in graph theory, we refer them to [26].

Definition 1. The degree of a node is defined as the number of edges connected with the node, and a node who has $k$ edges is called a degree- $k$ node.

Definition 2. An acyclic graph is a forest consisting of one or multiple trees, where each tree represents a connected component in the graph.

Proposition 2. A tree with $n$ nodes has $n-1$ edges. 
Proposition 3. A tree with $n \geq 2$ nodes has at least two degree-1 nodes.

Proposition 4. For an undirected tree, we can arbitrarily select a node as the root of the tree and each edge in the tree can be assigned a direction towards (or away from) the root node, which turns the tree into a directed rooted tree.

\section{Solution to Least Beam Collision Scheduling PROBLEM}

Assume that the edge pruning has been performed over graph $G$ and thus Proposition 1 holds. In searching the global minimum solution to the problem (7), we first give a lower bound to the number of beam collisions $n_{c}$. Then we prove that this lower bound is exactly the global minimum solution by certifying that this lower bound can be reached. Accordingly, the LBC algorithm is proposed to recursively acquire the schedule which reaches the minimum value of $n_{c}$.

\section{A. Overweight Edges and Lower Bound of $n_{c}$}

We define an edge $e_{k, k^{\prime}}$ as an overweight edge in a $(K, N)$ problem, if $\bar{d}_{k \rightarrow k^{\prime}}+\bar{d}_{k^{\prime} \rightarrow k} \geq N$. The set of all overweight edges in $G$ is denoted as $E_{o}$, which can be expressed as

$$
E_{o}=\left\{e_{k, k^{\prime}} \in E \mid \bar{d}_{k \rightarrow k^{\prime}}+\bar{d}_{k^{\prime} \rightarrow k} \geq N\right\} .
$$

An overweight edge $e_{k, k^{\prime}}$ indicates that cell $k$ and $k^{\prime}$ use the beams contained in $e_{k, k^{\prime}}$ at $\bar{d}_{k \rightarrow k^{\prime}}+\bar{d}_{k^{\prime} \rightarrow k} \geq N$ time slots in total. Intuitively, there are inevitably $\bar{d}_{k \rightarrow k^{\prime}}+\bar{d}_{k^{\prime} \rightarrow k}-N$ time slots with beam collision between two cells (if $\bar{d}_{k \rightarrow k^{\prime}}+$ $\bar{d}_{k^{\prime} \rightarrow k}=N$, there could be no beam collision). By counting all the inevitable beam collisions for each overweight edge, we can derive the following lemma which gives a lower bound of $n_{c}$.

Lemma 1. For a $(K, N)$ problem,

$$
n_{c} \geq \sum_{e_{k, k^{\prime}} \in E_{o}}\left(\bar{d}_{k \rightarrow k^{\prime}}+\bar{d}_{k^{\prime} \rightarrow k}-N\right) .
$$

Proof: See Appendix A.

Clearly, we need to prove that this lower bound is reachable.

\section{B. Subgraph of Overweight Edges}

We start from the subgraph $G_{o}=\left\{V_{o}, E_{o}\right\}$ formed by all the overweight edges in $E_{o}$, where $V_{o}$ is the set of the nodes connected by overweight edges. First consider that $G_{O}$ has circles, and we have the following lemma.

Lemma 2. For a $(K, N)$ problem, if its subgraph $G_{o}$ formed by the overweight edges has circles, the $(K, N)$ problem can be reduced to a smaller $\left(K_{0}, N\right)$ problem with $K_{0}<K$ which imposes the same number of beam collisions as the original problem. Furthermore, the subgraph of overweight edges for this smaller $\left(K_{0}, N\right)$ problem is acyclic.

Proof. See Appendix B.

From (24) in the proof, it can be seen that the appearance of a cycle in $G_{O}$ is an extremely peculiar event. According to Lemma 2, in proving the reachability of the lower bound (9), we only need to consider the case where $G_{o}$ is acyclic.

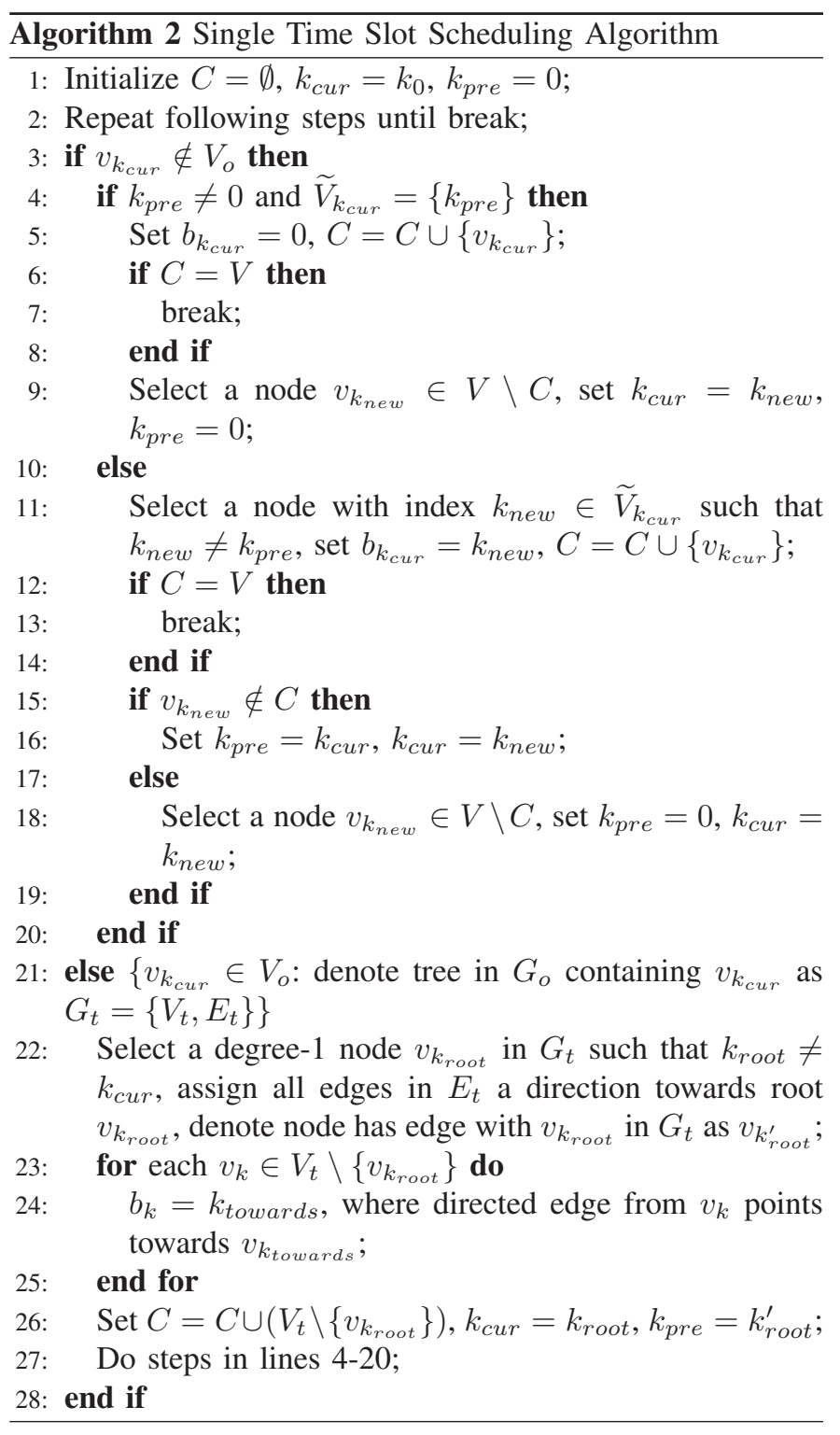

\section{Single Time Slot Scheduling}

From Lemma 2, we can consider the generic $(K, N)$ problem whose $G_{O}$ is acyclic. Since $G_{O}$ is acyclic, it is a forest. First, we introduce the definition of recursion condition.

Definition 3. A $(K, N)$ problem is said to meet the recursion condition if for every cell $k$, all its class demands are less than $N$, i.e.,

$$
\bar{d}_{k \rightarrow k^{\prime}}<N, 1 \leq k \leq K, \forall k^{\prime} \in \widetilde{V}_{k} \cup\{0\} .
$$

Any class demand must be met the constraint $\bar{d}_{k \rightarrow k^{\prime}} \leq N$. The recursion condition rules out the case of $\bar{d}_{k \rightarrow k^{\prime}}=N$. For a $(K, N)$ problem whose $G_{O}$ is acyclic, if the recursion condition (10) is met, we can apply Algorithm 2 to schedule a single time slot for the graph $G$. In Algorithm 2, $C$ is the set of the cells which has already been scheduled and it is initialized as $C=\emptyset$. A node $v_{k_{0}}$ is selected from $V$ arbitrarily as the first node considered and we use $k_{c u r}$ to represent the current node, which is initialized to be $k_{c u r}=k_{0}$. The algorithm also initializes an index $k_{\text {pre }}$ to $k_{\text {pre }}=0$. The index $k_{\text {pre }}$ is used 


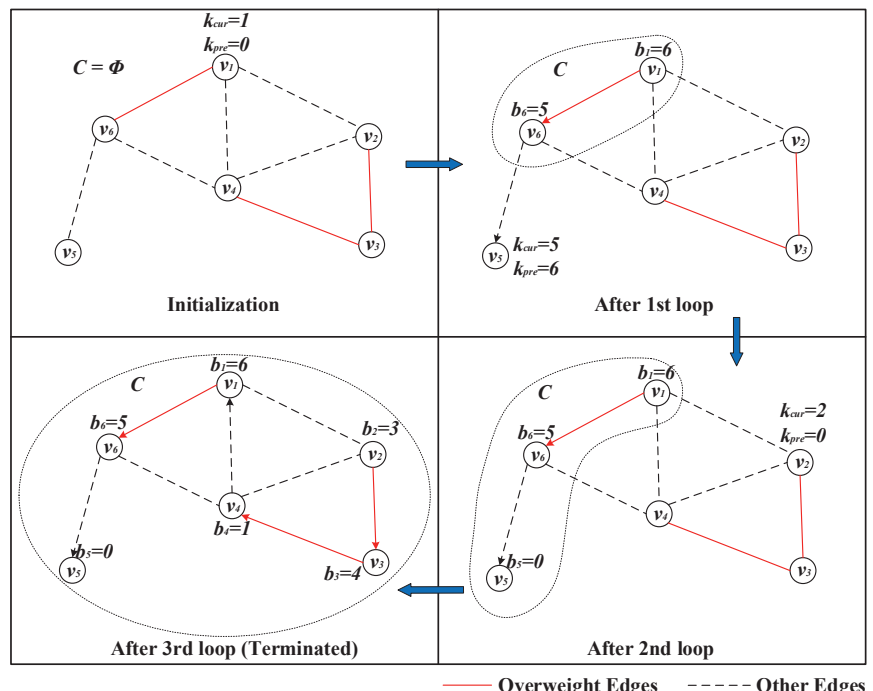

Fig. 3. An example of Algorithm 2 with $K=6$ cells. After initialization, the algorithms executes 3 loops and is terminated when $C=V$. The arrow from one node pointing towards another node indicates that the beam used by the source node may interfere with the destination node of the arrow.

to record the node in the previous step which uses a beam that may interfere with the current node, and if the previous node does not interfere with the current node, we set $k_{\text {pre }}=0$. Generally, the procedure of Algorithm 2 is to traverse all the $K$ nodes in $G$, which starts from $C=\emptyset$ and extends $C$ in each loop until $C=V$. Since Algorithm 2 terminates with $C=V$, every cell in the graph is scheduled. Furthermore, no beam collision occurs for this single time slot scheduling, which will be proved in Lemma 3.

An example is given in Fig. 3 to explain the actions of Algorithm 2. First note that all the overweight edges form the forest $G_{o}$ which consists of two trees.

Initialization. In the initialization of the algorithm, we select node $v_{1}$ as the first traversed node.

First loop. Since node $v_{1} \in G_{O}$ and is contained in tree $\left\{\left\{v_{1}, v_{6}\right\},\left\{e_{1,6}\right\}\right\}$, we select $v_{6}$ as the root of the tree, assign edge $e_{1,6}$ a direction towards the root, and set $b_{1}=6$ (lines 22-25). Next we consider the node $v_{6}$ with $k_{\text {pre }}=1$ and $C=\left\{v_{1}\right\}$, and try to find the next considered node (lines 26-27). Since $\widetilde{V}_{6}=\{1,4,5\}$, we can select $v_{5}$ as the next considered node, and thus we set $b_{6}=5, k_{\text {cur }}=5, k_{\text {pre }}=6$ and $C=\left\{v_{1}, v_{6}\right\}$ (lines 11-19).

Second loop. For the second loop, $v_{5}$ is the currently considered node and lines 5-9 are executed. We set $b_{5}=0$, $C=\left\{v_{1}, v_{5}, v_{6}\right\}$, and arbitrarily select a node from $V \backslash C=$ $\left\{v_{2}, v_{3}, v_{4}\right\}$, e.g., node $v_{2}$. Then, $k_{\text {cur }}$ is set to 2 and $k_{\text {pre }}=0$. Third loop. Since $v_{2} \in G_{o}$, the third loop is similar to the first loop, where $v_{4}$ is chosen to be the root of tree $\left\{\left\{v_{2}, v_{3}, v_{4}\right\},\left\{e_{2,3}, e_{3,4}\right\}\right\}$ and thus we set $b_{2}=3, b_{3}=4$, $C=\left\{v_{1}, v_{2}, v_{3}, v_{5}, v_{6}\right\}$. Then, we arbitrarily select a node among $\left\{v_{1}, v_{2}, v_{6}\right\}$, e.g., node $v_{1}$, and we set $b_{4}=1$ and $C=\left\{v_{1}, v_{2}, v_{3}, v_{4}, v_{5}, v_{6}\right\}$.

Termination. Since we have $C=V$ now, the algorithm is terminated. It can be seen that every cell is scheduled and no beam collision occurs.

For convenience, the schedule of the first time slot, i.e., the first column of $\boldsymbol{B}$, is denoted as $\boldsymbol{b} \in \mathbb{N}^{K \times 1}$. After the scheduling of the first time slot, we can denote the rest class demands of $M_{k \rightarrow k^{\prime}}$ as $\bar{d}_{k \rightarrow k^{\prime}}$, which can be expressed as

$$
\bar{d}_{k \rightarrow k^{\prime}}=\left\{\begin{array}{cc}
\bar{d}_{k \rightarrow k^{\prime}}-1, & \text { if } b_{k}=k^{\prime}, \\
\bar{d}_{k \rightarrow k^{\prime}}, & \text { if } b_{k} \neq k^{\prime},
\end{array}\right.
$$

where $b_{k}$ represents the $k$ th entry in $\boldsymbol{b}$.

Lemma 3. For a generic $(K, N)$ problem whose $G_{o}$ is acyclic, if the recursion condition (10) is met, we can acquire the schedule of the first time slot $\boldsymbol{b}$ using Algorithm 2, which induces no beam collision. Furthermore, the schedule of the rest $N-1$ time slots is a $(K, N-1)$ problem and this $(K, N-1)$ problem has the same lower bound of beam collisions as the original $(K, N-1)$, that is,

$$
\begin{aligned}
& \sum_{e_{k, k^{\prime}} \in E_{o}^{\prime}}\left(\bar{d}_{k \rightarrow k^{\prime}}+\bar{d}^{\prime}{ }_{k^{\prime} \rightarrow k}-(N-1)\right) \\
= & \sum_{e_{k, k^{\prime}} \in E_{o}}\left(\bar{d}_{k \rightarrow k^{\prime}}+\bar{d}_{k^{\prime} \rightarrow k}-N\right),
\end{aligned}
$$

where $E_{o}^{\prime}=\left\{e_{k, k^{\prime}} \mid \bar{d}^{\prime}{ }_{k \rightarrow k^{\prime}}+\bar{d}^{\prime}{ }_{k^{\prime} \rightarrow k} \geq(N-1)\right\}$ represents the overweight edge set of the 'reduced' $(K, N-1)$ problem.

Proof. See Appendix C.

\section{Global Minimum Solution}

We are now ready to prove that the lower bound given by Lemma 1 is reachable. In fact, based on Lemmas 2 and 3, we can prove that this lower bound is exactly the global minimum solution to the $(K, N)$ problem (7), which is stated in the following theorem.

Theorem 1. For the $(K, N)$ problem (7), the global minimum solution is given by

$$
n_{c}^{\star}=\sum_{e_{k, k^{\prime}} \in E_{o}}\left(\bar{d}_{k \rightarrow k^{\prime}}+\bar{d}_{k^{\prime} \rightarrow k}-N\right) .
$$

Proof. See Appendix D.

Moreover, from Theorem 1, the following corollary is obvious.

Corollary 1. If $E_{o}=\emptyset, n_{c}^{\star}=0$.

Based on the proof of Theorem 1, the LBC algorithm is proposed in Algorithm 3 for beam scheduling which guarantees to reach the minimum number of beam collisions $n_{c}^{\star}$.

The LBC algorithm is inherently recursive. For cases 3,4 and 5, either $K$ or $N$ is reduced, and then the LBC algorithm for a smaller problem is called. When either $K$ or $N$ is reduced to 1 , we have case 1 or case 2 , which can be scheduled easily.

We now analyze the computational complexity of the proposed LBC algorithm. In graph $G$, since each cell only has edges with its geographically neighboring cells, the degree of each node is limited. Thus we assume that the degree of each node is not larger than a constant $c$. Then, we consider the worst case where case 5 appears in every recursion with no reduction of $K$, and $N$ recursions are required. In each recursion, edge pruning and finding overweight edges both induce a computational complexity on the order of $\mathrm{O}(c K)$. Because there are at most $K$ overweight edges, the 


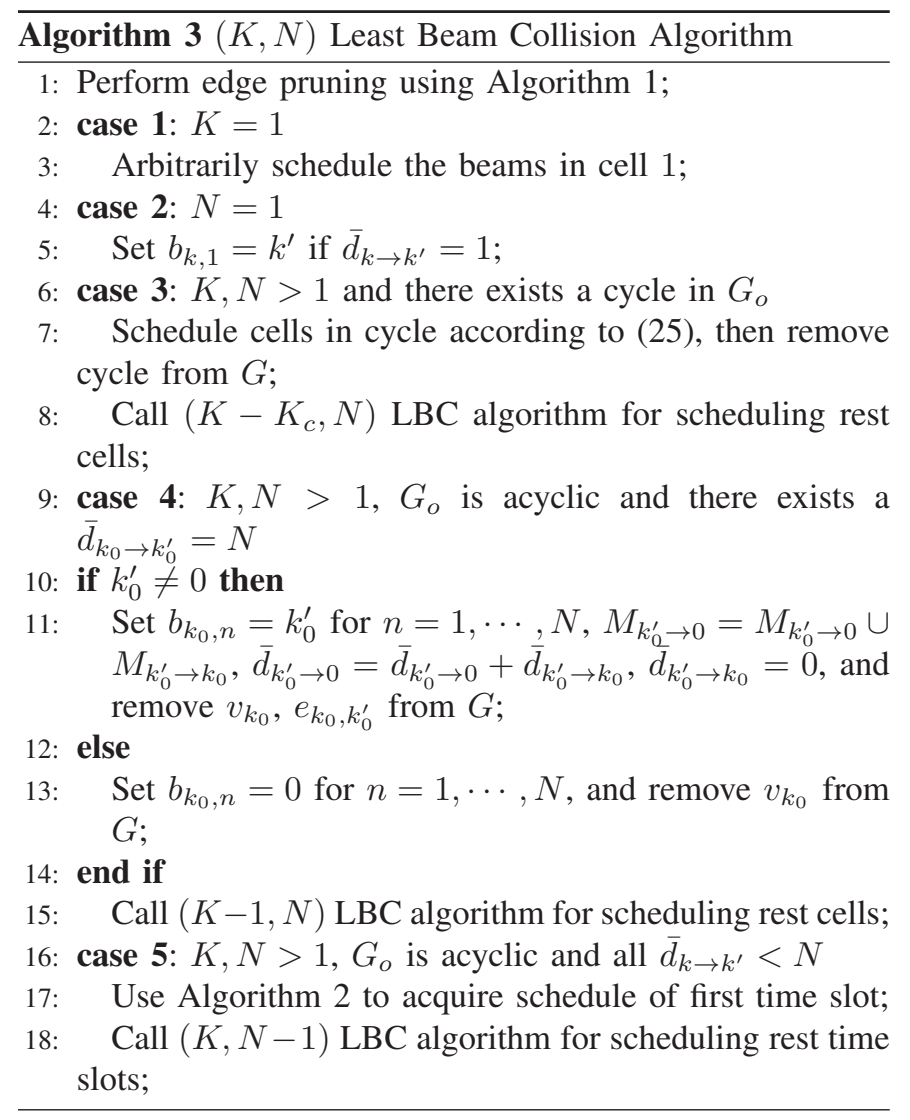

computational complexity of finding the trees in $G_{O}$ and judging whether there exists a cycle in $G_{O}$ is $\mathrm{O}\left(K^{2}\right)$. Next, in the single time slot scheduling, it can be seen that the complexity of adding each node into $C$ and finding the next considered node does not exceed $\mathrm{O}(K)$, and the complexity of single time slot scheduling is also $\mathrm{O}\left(K^{2}\right)$. Therefore, the computational complexity of one recursion for one time slot is $\mathrm{O}\left(c K+K^{2}\right)=\mathrm{O}\left(K^{2}\right)$ and the total computational complexity of Algorithm 3 is $\mathrm{O}\left(N K^{2}\right)$.

\section{E. Extension to Weighted Edges}

Due to the uncertainties of the practical environment between two neighboring cells imposed by potential scatters or obstacles, the performance loss caused by the beam collisions corresponding to different edges might be different. Therefore, a promising extension of our scheduling problem is to assign different weights to the edges according to the level of interference. It is fortunate that the scheduling problem after assigning weights would still have the same optimal beam scheduling solution as the original problem. Hence, the LBC algorithm can still be adopted to find the optimal solution.

We denote the weight assigned to edge $e_{k, k^{\prime}}$ as $w_{k, k^{\prime}}$, and the number of weighted beam collisions $n_{c, n e w}$ is expressed as

$$
n_{c, n e w}=\sum_{n=1}^{N} \sum_{e_{k, k^{\prime}} \in E} w_{k, k^{\prime}}\left(\delta\left(b_{k, n}, k^{\prime}\right) \delta\left(b_{k^{\prime}, n}, k\right)\right) .
$$
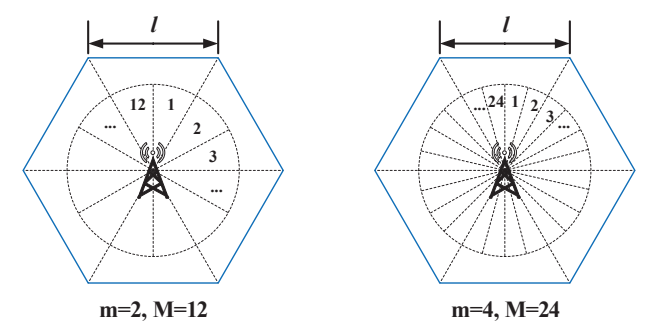

Fig. 4. Beam pattern in a cell. The number of beams in each $60^{\circ}$ sector is $m=2,4$ and the total number of beams is $M=12,24$.

On the one hand, similar to the proof of Lemma 1, by counting the inevitable weighted beam collisions caused by the edges in $E_{o}$, the lower bound of $n_{c, \text { new }}$ can be readily shown to be

$$
n_{c, \text { new }} \geq \sum_{e_{k, k^{\prime}} \in E_{o}} w_{k, k^{\prime}}\left(\bar{d}_{k \rightarrow k^{\prime}}+\bar{d}_{k^{\prime} \rightarrow k}-N\right) .
$$

On the other hand, since the optimal scheduling for the original problem (7) only causes the inevitable beam collisions in $E_{o}$, it also achieves the above lower bound of the number of weighted beam collisions. Therefore, this lower bound is reachable by applying our LBC scheduling to the original problem, and the global optimal number of weighted beam collisions can be expressed as

$$
n_{c, \text { new }}^{*}=\sum_{e_{k, k^{\prime}} \in E_{o}} w_{k, k^{\prime}}\left(\bar{d}_{k \rightarrow k^{\prime}}+\bar{d}_{k^{\prime} \rightarrow k}-N\right) .
$$

\section{Simulation Results}

Monte Carlo simulations are conducted to evaluate the performance of our proposed beam scheduling, in terms of the number of beam collisions incurred, cumulative distribution function (CDF) of SINR, and network sum rate. Since there has been no state-of-the-art research on mmWave ICI considering the different time-domain service demands of beams, the performance of the random scheduling with no cell coordination, the muting protocol [23], [24], and the ideal interference-free scheduling is simulated as benchmark counterparts.

\section{A. Simulation Setup}

We represent each cell by a regular hexagon region as shown in Fig. 4. Each cell is divided into six $60^{\circ}$ sectors and the number of beams in a sector is $m$. In our simulation, we set $m=2$ and 4 , and hence the total numbers of beams in two cells are $M=12$ and 24, respectively. Besides, we set $G_{\max }=10 \mathrm{~dB}$ if $m=2$, and $G_{\max }=13 \mathrm{~dB}$ if $m=4$, while $G_{\min }=0$ for both $m=2$ and 4 . The length of each side of regular hexagon cell is denoted as $l$.

As shown in Fig. 5, two scenarios are set up with 7 and 12 cells in the network, respectively. The beam classification is based on $60^{\circ}$ sector. For two neighboring cells, the beams in two adjacent $60^{\circ}$ sectors are filled with same patterns (the same color in the figure), and they form an edge connecting two neighboring cells. Accordingly, the graph models of the two scenarios are derived as shown in the figure. 
The 3GPP urban macro path loss model [27] is employed. The path losses of line-of-sight (LOS) and non-LOS (NLOS) paths are expressed respectively by

$$
\begin{aligned}
\mathcal{L}_{\mathrm{LOS}}(\mathrm{dB}) & =32.4+20 \log _{10}(d)+20 \log _{10}\left(f_{c}\right), \\
\mathcal{L}_{\mathrm{NLOS}}(\mathrm{dB}) & =13.54+39.08 \log _{10}(d)+20 \log _{10}\left(f_{c}\right),
\end{aligned}
$$

where $d$ represents the distance and $f_{c}$ is carrier frequency, which is set to $28 \mathrm{GHz}$. The probability of LOS path is given by

$$
\operatorname{Pr}_{\mathrm{LOS}}=\frac{18}{d}+\exp \left(-\frac{d}{36}\left(1-\frac{18}{d}\right)\right) .
$$

This model could take the effects of possible blockage and reflection into account.

The demands of beams are randomly generated but satisfying the constraint (2), and each beam is assumed to serve a user with the corresponding demand, where we set $N=30$. Since our objective is to evaluate the effect of ICI, we focus on the performance of cell-edge users, and hence the worst case is assumed where all users are considered at cell edge.

\section{B. Average Beam Collisions Comparison}

The average numbers of beam collision over 1000 simulations for scenarios A and B are shown in Fig. 6. In this simulation, we do not distinguish the two beam patterns. This is because the beam collision event is defined at class level, and the graph models of the two beam patterns are essentially the same after beam classification.

It can be seen that the average numbers of beam collisions under our LBC scheduling in both scenarios are substantially lower than those of the random scheduling in orders of magnitude. Since the LBC algorithm is capable of acquiring the minimum number of beam collisions, the average number of beam collisions under LBC scheduling can be extremely low, close to 0 . This implies that the number of beam collisions in LBC scheduling is 0 in most cases, where the strong ICI between every pair of neighboring cells can be completely eliminated.

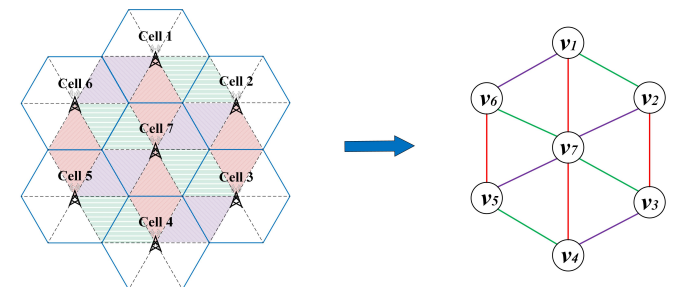

(a) Scenario A

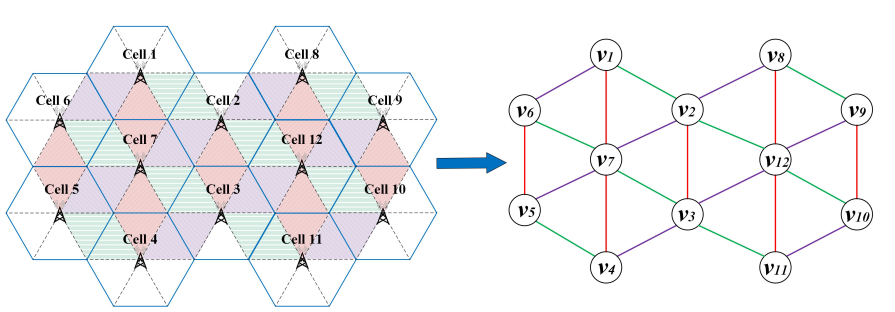

(b) Scenario B

Fig. 5. Networks with 7 cells (scenario A) and 12 cells (scenario B). The beams are classified by $60^{\circ}$ sectors, where two adjacent $60^{\circ}$ sectors of neighboring cells are filled with same pattern, which represents the beams that may cause ICI to each other, and they form an edge in the graph.
This results for the LBC scheduling actually agree with Corollary 1 . Because the class demands are randomly generated in each cell, the probability of the appearance of an overweight edge is very low. Therefore, we have $E_{o}=\emptyset$ in most cases. According to Corollary 1, the number of beam collisions is 0 when $E_{o}=\emptyset$. As a result, the number of average beam collisions achieved by the LBC scheduling would be nearly 0 . Additionally, the numbers of beam collisions achieved by the LBC scheduling are verified to match the minimum beam collision solution $n_{c}^{\star}$ given by Theorem 1 in all the 1000 simulations.

\section{SINR Comparison}

In the both scenarios, we set the transmission power of each cell to $20 \mathrm{dBm}$ and the noise power at user side to $-80 \mathrm{dBm}$, while the side length of each hexagon cell is chosen to be $l=50 \mathrm{~m}$. Then, we simulate the CDFs of SINR for all users in the network.

The CDF comparison between the SINR for the LBC and random scheduling is shown in Fig. 7. Observe that there are 3 regions in the CDF curve representing different levels of SINR. For example, for the senario of $m=2$ depicted in Fig. 7 (a), there is a low SINR region around $0 \mathrm{~dB}$, where the users suffer from the strong ICI from the closest neighboring cell and the power of ICI is almost equal to the received power from local cell. Also, a mid SINR region from $6 \mathrm{~dB}$ to $15 \mathrm{~dB}$ exists in Fig. 7 (a), where the ICI is from remote cells rather than the closest neighboring cell and, therefore, the SINR is relatively high. Finally, as shown in Fig. 7 (a), the majority of users lies in the highest SINR region from $15 \mathrm{~dB}$ to $16 \mathrm{~dB}$, where the users almost suffer from no ICI, which also indicates that no beam from other cells interferes with the users in this region.

Comparing the CDF of the SINR under LBC scheduling to that of the random scheduling, we observe that the low SINR region is eliminated by our LBC scheduling, which can improve the transmission reliability significantly. Under the random scheduling, burst of low SINR could frequently appear in the time domain, leading to bursty data detection errors. As a result, a relatively high symbol error rate (SER) is induced, and causes frequent frame or packet loss events. An additional set of simulation quantifying the SER of QPSK based on the SINR CDF given by Fig. 7 is shown in Table I, where we assume that the interference signal is scrambled and can be treated as noise. It can be seen that the elimination of the low SINR region around $0 \mathrm{~dB}$ remarkably enhances

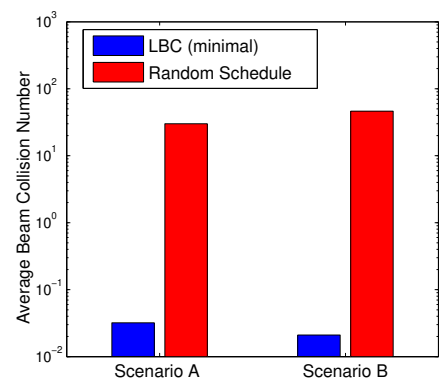

Fig. 6. Comparison of average beam collisions for LBC scheduling and random scheduling. 

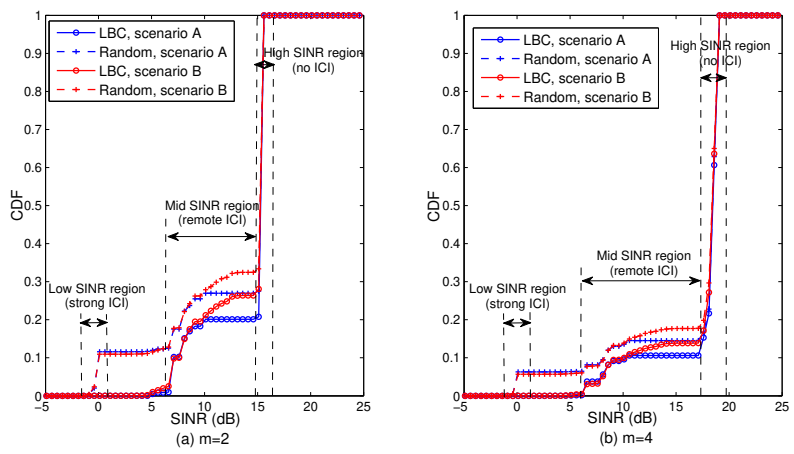

Fig. 7. CDFs of SINR of users in the network with $l=50 \mathrm{~m}$. (a) Scenari A $m=2$, and (b) Scenario B $m=4$.

$$
\text { TABLE I }
$$

SER Comparison between Random Scheduling and LBC Scheduling

\begin{tabular}{|c|c|c|c|c|}
\hline \multirow{2}{*}{} & \multicolumn{2}{|c|}{ Scenario A } & \multicolumn{2}{c|}{ Scenario B } \\
\cline { 2 - 5 } & $m=2$ & $m=4$ & $m=2$ & $m=4$ \\
\hline Random & $6.53 \mathrm{e}-2$ & $3.90 \mathrm{e}-2$ & $6.48 \mathrm{e}-2$ & $3.74 \mathrm{e}-2$ \\
\hline LBC & $7.00 \mathrm{e}-5$ & $2.60 \mathrm{e}-6$ & $2.30 \mathrm{e}-5$ & $1.90 \mathrm{e}-6$ \\
\hline
\end{tabular}

the transmission reliability of LBC scheduling compared with random scheduling.

Furthermore, the proportions of users in the mid SINR region of LBC scheduling and random scheduling are close. Consequently, the proportion of users in the high SINR region suffering no ICI for the LBC scheduling is clearly larger than that for the random scheduling.

Generally, less users are in the low SINR region for the $m=4$ scenario than for the $m=2$ scenario, and similarly less users are in the mid SINR region for the $m=4$ scenario than for the $m=2$ scenario. This is because the beamwidth is narrower when $m=4$, and the probability of the occurrence of ICI is also smaller.

\section{Network Sum Rate Comparison}

Next we compare the network's sum rate achieved by our LBC scheduling to those of the random scheduling, the muting protocol and the ideal interference-free scheduling. The system bandwidth is set to $500 \mathrm{MHz}$. For the interference-free scheduling, we assume that the beams are scheduled in the same manner as the random scheduling but the ICI powers

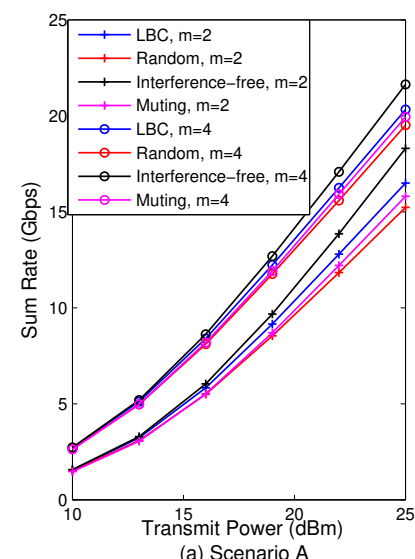

(a) Scenario $A$

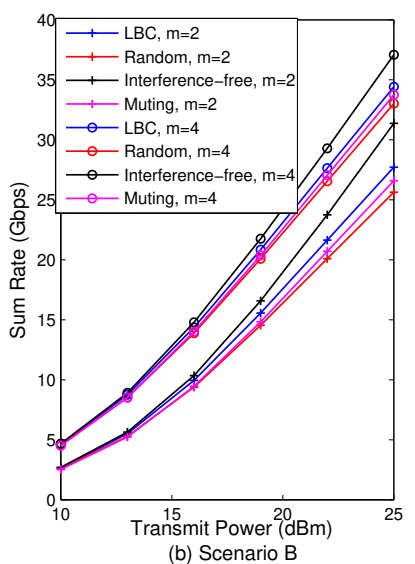

(b) Scenario B
Fig. 8. Network sum rates with $l=50 \mathrm{~m}, m=2$ and 4. (a) Scenario A, and (b) Scenario B.
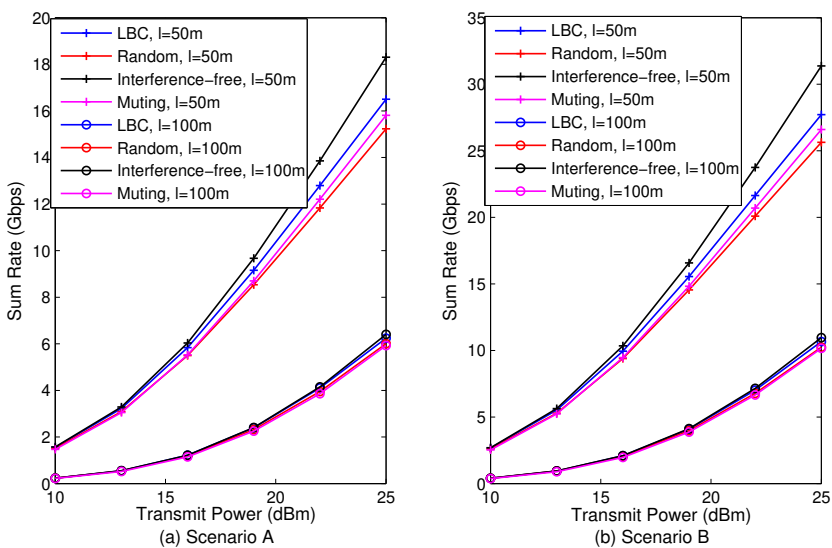

Fig. 9. Network sum rates with $m=2, l=50 \mathrm{~m}$ and $l=100 \mathrm{~m}$. (a) Scenario A, and (b) Scenario B.

are all 0 . Therefore, the interference-free scheduling serves as an ideal upper bound of network sum rate.

The sum rate comparison of the four schedules with $l=$ $50 \mathrm{~m}, m=2$ and 4 is demonstrated in Fig. 8 for the both network scenarios. As expected, the sum rate performance of the LBC scheduling lies between the interference-free upper bound and the sum rate of the random scheduling. According to our results in Section $\mathrm{V}-\mathrm{C}$, the performance gain of the LBC scheduling over the random scheduling mainly results from the elimination of strong ICI. The performance gap between the LBC and the ideal interference-free scheduling can be attributed to remote ICI. Meanwhile, the sum rates achieved by the muting protocol under different parameters are less than that of LBC scheduling because of the muted time slots. However, since the strong ICI is avoided, the muting protocol outperforms the random scheduling. In addition, the information whether a certain beam causes strong ICI to a certain user is assumed to be known by the muting protocol, which indicates that the muting protocol requires more accurate CSI than our LBC scheduling.

The effect of different cell densities is further simulated by comparing the sum rates for the cases of $l=50 \mathrm{~m}$ and $l=100 \mathrm{~m}$, as shown in Fig. 9. It can be seen that when $l=100 \mathrm{~m}$, the sum rates of the four schedules are very close, which indicates that the effect of ICI is negligible. This result agrees with the conclusions given in [10]-[12], namely that the network becomes noise-limited when the cell density is low. When $l$ is reduced to $50 \mathrm{~m}$, while the transmission power and bandwidth both remain unchanged, the increase of cell density leads to a significant increase of sum rate for all four scheduling schemes. Meanwhile, the power of ICI also increases with the cell density and becomes an important factor which limits the network's performance, resulting in obvious performance gaps between the four scheduling schemes. In this case, our proposed LBC scheduling can be utilized to avoid the strong ICI between neighboring cells and hence to boost the sum rate as well.

\section{Conclusions}

In this paper, a new ICI avoidance method based on time-domain beam scheduling for mmWave cellular network has been proposed. Specifically, by classifying the beams 
according to the cells that they may interfere with, we have modeled the entire network as a graph and we have proposed to schedule the beams in each cell in time domain to minimize the number of beam collisions, which eliminates the strong ICI caused by neighboring cells as much as possible. Our proposed scheduling is fully transparent to users and, therefore, no CSI exchange is required between the users and the network in our scheduling. We have proved via graph theory that the proposed LBC scheduling algorithm guarantees to acquire the global minimum number of beam collisions. The theoretical results have also shown that the minimum number of beam collisions is 0 in most cases, which indicates that the strong ICI usually can be avoided completely. Simulation results have verified our theoretical analysis.

\section{APPENDIX}

\section{A. Proof of Lemma 1}

Proof: If $E_{o}=\emptyset$, Lemma 1 is equivalent to $n_{c} \geq 0$, which is apparently true.

If $E_{o} \neq \emptyset$, we arbitrarily consider an edge $e_{k, k^{\prime}} \in E_{o}$. Obviously, $\bar{d}_{k \rightarrow k^{\prime}}+\bar{d}_{k^{\prime} \rightarrow k} \geq N$. We denote $T_{k \rightarrow k^{\prime}}$ and $T_{k^{\prime} \rightarrow k}$ as the sets of time slots during which cell $k$ uses the beams in $M_{k \rightarrow k^{\prime}}$ and cell $k^{\prime}$ uses the beams in $M_{k^{\prime} \rightarrow k}$, respectively. Or equivalently, we can express

$$
T_{k \rightarrow k^{\prime}}=\left\{n \mid b_{k, n}=k^{\prime}\right\} \text { and } T_{k^{\prime} \rightarrow k}=\left\{n \mid b_{k^{\prime}, n}=k\right\},
$$

where we have $\left|T_{k \rightarrow k^{\prime}}\right|=\bar{d}_{k \rightarrow k^{\prime}}$ and $\left|T_{k^{\prime} \rightarrow k}\right|=$ $\bar{d}_{k^{\prime} \rightarrow k}$ owing to the service demand constraint. Note that $\delta\left(b_{k, n}, k^{\prime}\right) \delta\left(b_{k^{\prime}, n}, k\right)=1$ if and only if $n \in T_{k \rightarrow k^{\prime}} \cap T_{k^{\prime} \rightarrow k}$. Besides, we have $\left|T_{k \rightarrow k^{\prime}} \cup T_{k^{\prime} \rightarrow k}\right| \subset\{1, \cdots, N\}$. Hence,

$$
\begin{aligned}
& \sum_{n=1}^{N}\left(\delta\left(b_{k, n}, k^{\prime}\right) \delta\left(b_{k^{\prime}, n}, k\right)\right)=\left|T_{k \rightarrow k^{\prime}} \cap T_{k^{\prime} \rightarrow k}\right| \\
& =\left|T_{k \rightarrow k^{\prime}}\right|+\left|T_{k^{\prime} \rightarrow k}\right|-\left|T_{k \rightarrow k^{\prime}} \cup T_{k^{\prime} \rightarrow k}\right| \\
& \geq\left|T_{k \rightarrow k^{\prime}}\right|+\left|T_{k^{\prime} \rightarrow k}\right|-N=\bar{d}_{k \rightarrow k^{\prime}}+\bar{d}_{k^{\prime} \rightarrow k}-N .
\end{aligned}
$$

By substituting (18) into (4), we arrive at

$$
\begin{aligned}
n_{c} & =\sum_{n=1}^{N} \sum_{e_{k, k^{\prime}} \in E}\left(\delta\left(b_{k, n}, k^{\prime}\right) \delta\left(b_{k^{\prime}, n}, k\right)\right) \\
& =\sum_{e_{k, k^{\prime}} \in E} \sum_{n=1}^{N}\left(\delta\left(b_{k, n}, k^{\prime}\right) \delta\left(b_{k^{\prime}, n}, k\right)\right) \\
& \geq \sum_{e_{k, k^{\prime}} \in E_{o}} \sum_{n=1}^{N}\left(\delta\left(b_{k, n}, k^{\prime}\right) \delta\left(b_{k^{\prime}, n}, k\right)\right) \\
& \geq \sum_{e_{k, k^{\prime}} \in E_{o}}\left(\bar{d}_{k \rightarrow k^{\prime}}+\bar{d}_{k^{\prime} \rightarrow k}-N\right) .
\end{aligned}
$$

This completes the proof.

\section{B. Proof of Lemma 2}

Proof. Consider any circle of $G_{o}$ and denote this cycle as a subgraph $G_{c}^{1}=\left\{V_{c}^{1}, E_{c}^{1}\right\}$ of $G_{o}$, where $V_{c}^{1}$ and $E_{c}^{1}$ are the node set and the edge set of this cycle, respectively. The number of the nodes in $G_{c}^{1}$ is denoted as
$K_{c}^{1}$. Further denote $V_{c}^{1}=\left\{v_{1}, v_{2}, \cdots, v_{K_{c}^{1}}\right\}$ and $E_{c}^{1}=$ $\left\{e_{1,2}, e_{2,3}, \cdots, e_{K_{c}^{1}-1, K_{c}^{1}}, e_{K_{c}^{1}, 1}\right\}$. Since $G_{c}^{1}$ is a subgraph of $G_{o}$, the edges in $E_{c}^{1}$ are overweight edges, i.e., $E_{c}^{1} \subset E_{o}$. Therefore,

$$
\sum_{k=1}^{K_{c}^{1}}\left(\bar{d}_{k \rightarrow k+1}+\bar{d}_{k+1 \rightarrow k}\right) \geq \sum_{k=1}^{K_{c}^{1}} N=K_{c}^{1} N
$$

where for convenience we introduce the node $v_{K_{c}^{1}+1}$ which is actually $v_{1}$ in the cycle $G_{c}^{1}$, and hence $\bar{d}_{K_{c}^{1} \rightarrow K_{c}^{1}+1}=\bar{d}_{K_{c}^{1} \rightarrow 1}$ and $\bar{d}_{K_{c}^{1}+1 \rightarrow K_{c}^{1}}=\bar{d}_{1 \rightarrow K_{c}^{1}}$ in (20). On the other hand, since the class demands in each cell must satisfy (2), we have

$$
\begin{aligned}
\sum_{k=1}^{K_{c}^{1}}\left(\bar{d}_{k \rightarrow k+1}+\bar{d}_{k+1 \rightarrow k}\right) & =\sum_{k=1}^{K_{c}^{1}}\left(\bar{d}_{k \rightarrow k-1}+\bar{d}_{k \rightarrow k+1}\right) \\
& \leq \sum_{k=1}^{K_{c}^{1}} N=K_{c}^{1} N,
\end{aligned}
$$

where for convenience we introduce the node $v_{0}$ which is actually $v_{K_{c}^{1}}$ in the cycle $G_{c}$, and thus $\bar{d}_{1 \rightarrow 0}=\bar{d}_{1 \rightarrow K_{c}^{1}}$ in (21). Comparing (20) with (21), it can be seen that

$$
\sum_{k=1}^{K_{c}^{1}}\left(\bar{d}_{k \rightarrow k+1}+\bar{d}_{k+1 \rightarrow k}\right)=K_{c}^{1} N
$$

which indicates that the inequalities in (20) and (21) both become equality. Therefore, we have

$$
\bar{d}_{k \rightarrow k-1}+\bar{d}_{k \rightarrow k+1}=\bar{d}_{k \rightarrow k+1}+\bar{d}_{k+1 \rightarrow k}=N,
$$

for $k=1, \cdots, K_{c}^{1}$. From (23), we can derive

$$
\begin{aligned}
& \bar{d}_{1 \rightarrow 2}=\bar{d}_{2 \rightarrow 3}=\cdots=\bar{d}_{K_{c}^{1} \rightarrow 1}, \\
& \bar{d}_{2 \rightarrow 1}=\bar{d}_{3 \rightarrow 2}=\cdots=\bar{d}_{1 \rightarrow K_{c}^{1}}=N-\bar{d}_{1 \rightarrow 2} .
\end{aligned}
$$

Owing to (2) and (23), the class demands other than $\bar{d}_{k \rightarrow k-1}$ and $\bar{d}_{k \rightarrow k+1}$ of $v_{k} \in V_{c}^{1}$ are all 0 . Furthermore, if there exists an edge $e_{k, k^{\prime}}$ connecting $v_{k} \in V_{c}^{1}$ with a node $v_{k^{\prime}} \in V \backslash V_{c}^{1}$, Proposition 1 implies that the edge can be pruned because $\bar{d}_{k \rightarrow k^{\prime}}=0$. Thus, $G_{c}^{1}$ is an isolated subgraph in $G$. Based on (24), we can schedule the cells 1 to $K_{c}^{1}$ in $G_{c}^{1}$ as follow

$$
\begin{cases}b_{k, n}=k+1, & n=1, \cdots, \bar{d}_{1 \rightarrow 2}, k=1, \cdots, K_{c}^{1}, \\ b_{k, n}=k-1, & n=\bar{d}_{1 \rightarrow 2}+1, \cdots, N, k=1, \cdots, K_{c}^{1},\end{cases}
$$

where again similar to our previous notations, $b_{1, n}=0$ and $b_{K_{c}^{1}, n}=K_{c}^{1}+1$ in the cycle actually represent $b_{1, n}=K_{c}^{1}$ and $b_{K_{c}^{1}, n}=1$, respectively. It can be easily verified that the schedule (25) imposes 0 beam collision in $G_{c}^{1}$ over $N$ time slots. Consequently, due to the isolation between $V_{c}^{1}$ and $V \backslash V_{c}^{1}$, the schedule of the rest cells in $V \backslash V_{c}^{1}$, which is a $\left(K-K_{c}^{1}, N\right)$ problem, must imposes the same number of beam collisions as the original $(K, N)$ problem.

If $G_{c}^{1}$ is the only circle of $G_{O}$, the subgraph of overweight edges corresponding to the $\left(K-K_{c}^{1}, N\right)$ problem is obviously acyclic. Otherwise, let the original $G_{O}$ contain $N_{c}>1$ circles, each having $K_{c}^{n_{c}}$ nodes for $1 \leq n_{c} \leq N_{c}$. We can repeat the above procedure to the remaining $N_{c}-1$ circles. Consequently, we can reduce the $(K, N)$ problem 
to a smaller $\left(K-\sum_{n_{c}=1}^{N_{c}} K_{c}^{n_{c}}, N\right)$ problem, which imposes the same number of beam collisions as the original $(K, N)$ problem. Moreover, the subgraph of overweight edges for this small problem is acyclic.

\section{Proof of Lemma 3}

Proof. Since the recursion condition (10) holds, we have $\bar{d}_{k \rightarrow k^{\prime}} \leq N-1, k^{\prime} \in \widetilde{V}_{k} \cup\{0\}$ for every $k$. From (11), it is obvious that

$$
\sum_{k^{\prime} \in \widetilde{V}_{k} \cup\{0\}} \bar{d}_{k \rightarrow k^{\prime}}^{\prime}=N-1, k=1, \cdots, K .
$$

Hence, the rest problem is a $(K, N-1)$ problem for an arbitrary $\boldsymbol{b}$.

According to the recursion condition (10) and the constraint (2), there are at least two non-zero entries in $\left\{\bar{d}_{k \rightarrow k^{\prime}}\right\}_{k^{\prime} \in \widetilde{V}_{k} \cup\{0\}}$ for every $k$. If there is only one nonzero entry in $\left\{\tilde{d}_{k \rightarrow k^{\prime}}\right\}_{k^{\prime} \in \tilde{V}_{k}}$, then we must have $\bar{d}_{k \rightarrow 0}>0$. Otherwise, $\left\{\bar{d}_{k \rightarrow k^{\prime}}\right\}_{k^{\prime} \in \widetilde{V}_{k}}$ must have two or more non-zero entries, i.e., $\left|\widetilde{V}_{k}\right| \geq 2$. Therefore, we have the following proposition.

Proposition 5. If the recursion condition (10) holds, then for each cell $k$, either $\left|\widetilde{V}_{k}\right| \geq 2$, or $\left|\widetilde{V}_{k}\right|=1$ and $\bar{d}_{k \rightarrow 0}>0$, for each cell $k$.

In Algorithm 2, the following property can be clearly observed.

Proposition 6. At the beginning of each loop, $k_{\text {cur }} \notin C$ and no cell in $C$ schedules a beam to interfere with cell $k_{\text {cur }}$ except cell $k_{\text {pre }}$, i.e., $b_{k} \neq k_{\text {cur }}$ for $k \in C \backslash\left\{k_{\text {pre }}\right\}$.

We now prove that every loop of Algorithm 2 is feasible and no beam collision occurs after each loop. Since we initialize $C=\emptyset$, there is no beam collision at the beginning. Hence, we consider an arbitrary loop and assume that the algorithm is feasible and no beam collision occurs before this loop. All the possibilities of node $v_{k_{c u r}}$ are discussed as follow.

(i) $v_{k_{\text {cur }}} \notin V_{o}$.

(i.1) $k_{\text {pre }} \neq 0$ and $\widetilde{V}_{k_{\text {cur }}}=\left\{k_{\text {pre }}\right\}$ :

Since $\left|\widetilde{V}_{k_{\text {cur }}}\right|=1$, according to Proposition 5, we have $\bar{d}_{k_{\text {cur }} \rightarrow 0}>0$. Therefore, cell $k_{\text {cur }}$ can use a beam in $M_{k_{\text {cur }} \rightarrow 0}$, i.e., $b_{k_{c u r}}=0$ and no beam collision occurs.

(i.2) $k_{\text {pre }}=0$ or $\widetilde{V}_{k_{\text {cur }}} \neq\left\{k_{\text {pre }}\right\}$ :

According to Proposition 5, we have $\widetilde{V}_{k_{c u r}} \neq \emptyset$ in either case. If $k_{\text {pre }}=0$, then we can arbitrarily select a $k_{n e w} \in \widetilde{V}_{k_{c u r}}$ such that $k_{\text {new }} \neq k_{\text {pre. }}$. If $k_{\text {pre }} \neq 0$, then we have $\widetilde{V}_{k_{\text {cur }}} \neq\left\{k_{\text {pre }}\right\}$. On the other hand, cell $k_{\text {pre }}$ interferes with cell $k_{c u r}$ because of $k_{\text {pre }} \neq 0$, which indicates that cell $k_{\text {pre }}$ is a neighboring cell of cell $k_{\text {cur }}$, i.e., $k_{\text {pre }} \in \widetilde{V}_{k_{\text {cur }}}$. Combining $k_{\text {pre }} \in \widetilde{V}_{k_{c u r}}$ with $\widetilde{V}_{k_{\text {cur }}} \neq\left\{k_{\text {pre }}\right\}$, it can be seen that there must exist an entry in $\widetilde{V}_{k_{c u r}}$ other than $k_{\text {pre }}$. Therefore, we can select a $k_{\text {new }} \in \widetilde{V}_{k_{\text {cur }}}$ such that $k_{\text {new }} \neq k_{\text {pre }}$.

If $v_{k_{n e w}} \notin C$, it is apparent that no beam collision occurs because cell $k_{\text {new }}$ has not been scheduled yet. If $v_{k_{n e w}} \in C$, the beam collision between cell $k_{\text {new }}$ and cell $k_{\text {cur }}$ occurs only if $b_{k_{\text {new }}}=k_{\text {cur }}$. However, since $k_{\text {new }} \neq k_{\text {pre }}, b_{k_{\text {new }}}=k_{\text {cur }}$ contradicts Proposition 6. Therefore, we must have $b_{k_{n e w}} \neq$ $k_{c u r}$ and no beam collision occurs.

(ii) $v_{k_{c u r}} \in V_{o}$

Since $G_{O}$ is acyclic, it is a forest. We denote the tree in $G_{O}$ containing $v_{k_{\text {cur }}}$ as $G_{t}=\left\{V_{t}, E_{t}\right\}$. According to Proposition 3, there must exist a degree-1 node $v_{k_{\text {root }}}$ in $G_{t}$ such that $k_{\text {root }} \neq$ $k_{c u r}$. Then, according to Proposition 4, the procedure in lines 22-25 of Algorithm 2 is feasible to schedule the nodes in $V_{t} \backslash\left\{v_{k_{\text {root }}}\right\}$.

Note that, for every $v_{k} \in V_{t}$, we have $v_{k} \notin C$, otherwise $v_{k}$ would have already been scheduled in the previous loops, which results in $k_{\text {cur }} \in C$ and contradicts Proposition 6 . Therefore, the schedule of the nodes in $V_{t} \backslash\left\{v_{k_{\text {root }}}\right\}$ does not cause beam collision since they have not been scheduled. Since $v_{k_{\text {root }}}$ is a degree-1 node in $G_{t}$, the schedule of cell $k_{\text {root }}$ is equivalent to case (i) with $k_{c u r}=k_{\text {root }}$ and $k_{\text {pre }}=k_{\text {root }}^{\prime}$, where $k_{\text {root }}^{\prime}$ denotes the node having edge with $v_{k_{\text {root }}}$ in $G_{t}$.

So far, we have certified the feasibility of Algorithm 2 and proved that no beam collision occurs in the schedule of $\boldsymbol{b}$. Next, we prove that (12) is also satisfied.

In lines 22-25, according to Proposition 2 and 4, for every tree $G_{t}=\left\{V_{t}, E_{t}\right\}$ in $G_{o}$, all $\left|V_{t}\right|-1$ edges in $G_{t}$ are assigned a direction. Therefore, for every overweight edge $e_{k, k^{\prime}} \in E_{o}$, one of cell $k$ and cell $k^{\prime}$ uses the beam contained in $e_{k, k^{\prime}}$. Thus we have

$$
\bar{d}_{k \rightarrow k^{\prime}}^{\prime}+{\overline{d^{\prime}}}_{k^{\prime} \rightarrow k}=\bar{d}_{k \rightarrow k^{\prime}}+\bar{d}_{k^{\prime} \rightarrow k}-1,
$$

for every $e_{k, k^{\prime}} \in E_{o}$. This leads to

$$
\bar{d}_{k \rightarrow k^{\prime}}^{\prime}+\bar{d}_{k^{\prime} \rightarrow k}-(N-1)=\bar{d}_{k \rightarrow k^{\prime}}+\bar{d}_{k^{\prime} \rightarrow k}-N \geq 0,
$$

for every $e_{k, k^{\prime}} \in E_{o}$, which indicates that every overweight edge $e_{k, k^{\prime}} \in E_{o}$ is an overweight edge in the rest $(K, N-1)$ problem, i.e., $E_{o} \subset E_{o}^{\prime}$. On the other hand, if $E_{o}^{\prime} \backslash E_{o} \neq \emptyset$, for every edge $e_{k, k^{\prime}} \in E_{o}^{\prime} \backslash E_{o}$, we have

$$
\bar{d}_{k \rightarrow k^{\prime}}+\bar{d}_{k^{\prime} \rightarrow k}^{\prime}-(N-1) \geq 0, \bar{d}_{k \rightarrow k^{\prime}}+\bar{d}_{k^{\prime} \rightarrow k}-N<0 .
$$

Noting that $\bar{d}_{k \rightarrow k^{\prime}}+\bar{d}_{k^{\prime} \rightarrow k} \leq \bar{d}_{k \rightarrow k^{\prime}}+\bar{d}_{k^{\prime} \rightarrow k}$ due to (11), we can derive

$$
\bar{d}_{k \rightarrow k^{\prime}}+\bar{d}_{k^{\prime} \rightarrow k}^{\prime}=\bar{d}_{k \rightarrow k^{\prime}}+\bar{d}_{k^{\prime} \rightarrow k}=N-1,
$$

for every $e_{k, k^{\prime}} \in E_{o}^{\prime} \backslash E_{o}$. From (28) and (30), we arrive at

$$
\begin{aligned}
& \sum_{e_{k, k^{\prime}} \in E_{o}^{\prime}}\left(\bar{d}^{\prime}{ }_{k \rightarrow k^{\prime}}+\bar{d}_{k^{\prime} \rightarrow k}{ }^{\prime}-(N-1)\right) \\
= & \sum_{e_{k, k^{\prime}} \in E_{o}}\left(\bar{d}^{\prime}{ }_{k \rightarrow k^{\prime}}+\bar{d}_{k^{\prime} \rightarrow k}-(N-1)\right)+ \\
& \sum_{e_{k, k^{\prime}} \in E_{o}^{\prime} \backslash E_{o}}\left(\bar{d}_{k \rightarrow k^{\prime}}+\bar{d}_{k^{\prime} \rightarrow k}-(N-1)\right) \\
= & \sum_{e_{k, k^{\prime}} \in E_{o}}\left(\bar{d}_{k \rightarrow k^{\prime}}+\bar{d}_{k^{\prime} \rightarrow k}-N\right) .
\end{aligned}
$$

This completes the proof. 


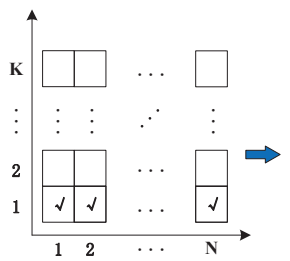

Step 1: prove $K=1$ case

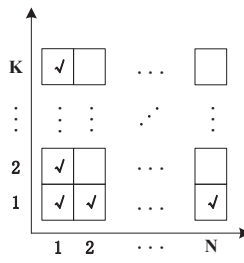

Step 2: prove $\mathbf{N}=1$ case

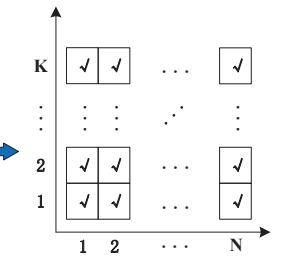

Step 3: prove general $(\mathbf{K}, \mathbf{N})$ case
Fig. 10. Three steps of the two-dimensional induction on $K$ and $N$ to prove Theorem. 1.

\section{Proof of Theorem 1}

Proof. Based on Lemma 1, we only have to prove that the lower bound is reachable. A two-dimensional induction on $K$ and $N$ is employed in the proof. The whole proof contains three steps as depicted in Fig. 10.

Firstly, we consider the $K=1$ case, where $N$ can be arbitrary. Since there is only a single cell, no beam collision occurs for any scheduling. On the other hand, no edge exists in $G$, so $E_{o}=\emptyset$ and we have

$$
n_{c}^{\star}=0=\sum_{e_{k, k^{\prime}} \in E_{o}}\left(\bar{d}_{k \rightarrow k^{\prime}}+\bar{d}_{k^{\prime} \rightarrow k}-N\right) .
$$

Therefore, Theorem 1 holds for $K=1$.

Then, we consider the $N=1$ case, where $K$ can be arbitrary. Due to (2), for each cell $k$, there is only one entry in $\left\{\bar{d}_{k \rightarrow k^{\prime}}\right\}_{k^{\prime} \in \widetilde{V}_{k} \cup\{0\}}$ which is 1 , and the other entries are all 0 . Therefore, there is only one feasible scheduling, where each cell uses the beam in the only class whose class demand is 1. In this case, according to Proposition 1, an edge $e_{k, k^{\prime}} \in E$ if and only if $\bar{d}_{k \rightarrow k^{\prime}}=\bar{d}_{k^{\prime} \rightarrow k}=1$, which also indicates that $\bar{d}_{k \rightarrow k^{\prime}}+\bar{d}_{k^{\prime} \rightarrow k}-N=1$ for each $e_{k, k^{\prime}} \in E$ and thus $E=E_{o}$. Therefore, we have

$$
\begin{aligned}
n_{c}^{\star} & =\sum_{e_{k, k^{\prime}} \in E}\left(\delta\left(b_{k, 1}, k^{\prime}\right) \delta\left(b_{k^{\prime}, 1}, k\right)\right)=\sum_{e_{k, k^{\prime}} \in E_{o}} 1 \\
& =\sum_{e_{k, k^{\prime}} \in E_{o}}\left(\bar{d}_{k \rightarrow k^{\prime}}+\bar{d}_{k^{\prime} \rightarrow k}-N\right),
\end{aligned}
$$

that is, Theorem 1 holds for $N=1$.

Since we have proved the theorem for $K=1$ case and the $N=1$ case, to prove the general case of $K=K_{0}, N=N_{0}$, we can assume that Theorem 1 holds for all $K \leq K_{0}, N \leq N_{0}$ cases, except for the $K=K_{0}, N=N_{0}$ case. Then, we will prove that Theorem 1 holds for the $K=K_{0}, N=N_{0}$ case. There are three possibilities as discussed below.

(i) There exists a cycle in $G_{o}$.

According to the proof of Lemma 2, by denoting the cycle as a subgraph $G_{c}=\left\{V_{c}, E_{c}\right\}$, where $V_{c}=\left\{v_{1}, \cdots, v_{K_{c}}\right\}$, the nodes in $V_{c}$ can be scheduled according to (25) with no beam collision. Moreover, since $G_{c}$ is isolated from $G$, the schedule of the rest nodes is a $\left(K-K_{c}, N\right)$ problem.

Denote the rest graph as $G^{\prime}=\left\{V \backslash V_{c}, E \backslash E_{c}\right\}$, where the class demands in each cell remain unchanged. Denoting the overweight edge set of the original graph $G$ by $E_{o}$, then the overweight edge set of $G^{\prime}$ is $E_{o} \backslash E_{c}$. According to our inductive assumption on $K$, the residual $\left(K-K_{c}, N\right)$ problem can be solved with the minimum number of beam collisions

$$
n_{\text {res }}^{\star}=\sum_{e_{k, k^{\prime}} \in E_{o} \backslash E_{c}}\left(\bar{d}_{k \rightarrow k^{\prime}}+\bar{d}_{k^{\prime} \rightarrow k}-N\right) .
$$

Therefore, the total number of beam collisions is $n_{\text {res }}^{\star}$ because the schedule for $G_{c}$ has no beam collision. On the other hand, according to (23), we have

$$
\bar{d}_{k \rightarrow k^{\prime}}+\bar{d}_{k^{\prime} \rightarrow k}-N=0, \forall e_{k, k^{\prime}} \in E_{c} .
$$

Hence, the minimum number of beam collisions given by Theorem 1

$$
\begin{aligned}
n_{c}^{\star} & =\sum_{e_{k, k^{\prime}} \in E_{o}}\left(\bar{d}_{k \rightarrow k^{\prime}}+\bar{d}_{k^{\prime} \rightarrow k}-N\right) \\
& =\sum_{e_{k, k^{\prime}} \in E_{o} \backslash E_{c}}\left(\bar{d}_{k \rightarrow k^{\prime}}+\bar{d}_{k^{\prime} \rightarrow k}-N\right) \\
& +\sum_{e_{k, k^{\prime}} \in E_{c}}\left(\bar{d}_{k \rightarrow k^{\prime}}+\bar{d}_{k^{\prime} \rightarrow k}-N\right)=n_{\text {res }}^{\star}
\end{aligned}
$$

is reached by the schedule.

(ii) $G_{o}$ is acyclic, and there exists a $\bar{d}_{k_{0} \rightarrow k_{0}^{\prime}}=N$.

According to (2), all the class demands other than $\bar{d}_{k_{0} \rightarrow k_{0}^{\prime}}$ of cell $k_{0}$ are 0 .

If $k_{0}^{\prime} \neq 0$, according to Proposition 1 , node $v_{k_{0}}$ only has an edge connected with $v_{k_{0}^{\prime}}$. Therefore, the schedule of cell $k_{0}$ must be $b_{k_{0}, n}=k_{0}^{\prime}, 1 \leq n \leq N$, and causes $\bar{d}_{k_{0}^{\prime} \rightarrow k_{0}}$ beam collisions. Note that edge $e_{k_{0}, k_{0}^{\prime}}$ is an overweight edge, since $\bar{d}_{k_{0} \rightarrow k_{0}^{\prime}}+\bar{d}_{k_{0}^{\prime} \rightarrow k_{0}} \geq N$.

Then, we remove node $v_{k_{0}}$ and edge $e_{k_{0}, k_{0}^{\prime}}$. Since the beams in class $M_{k_{0}^{\prime} \rightarrow k_{0}}$ do not cause beam collision after the removal of $v_{k_{0}}$, we can add these beams into the interference-free class, i.e., $M_{k_{0}^{\prime} \rightarrow 0}=M_{k_{0}^{\prime} \rightarrow 0} \cup M_{k_{0}^{\prime} \rightarrow k_{0}}, \bar{d}_{k_{0}^{\prime} \rightarrow 0}=\bar{d}_{k_{0}^{\prime} \rightarrow 0}+\bar{d}_{k_{0}^{\prime} \rightarrow k_{0}}$. The rest scheduling problem is a $(K-1, N)$ problem with graph $G^{\prime}=\left\{V \backslash\left\{v_{k_{0}}\right\}, E \backslash\left\{e_{k_{0}, k_{0}^{\prime}}\right\}\right\}$. According to our inductive assumption on $K$, this $(K-1, N)$ problem can be solved with the minimum number of beam collisions

$$
n_{\text {res }}^{\star}=\sum_{e_{k, k^{\prime}} \in E_{o} \backslash\left\{e_{k_{0}, k_{0}^{\prime}}\right\}}\left(\bar{d}_{k \rightarrow k^{\prime}}+\bar{d}_{k^{\prime} \rightarrow k}-N\right) .
$$

Therefore, the total number of beam collisions is $n_{\text {res }}^{\star}+\bar{d}_{k_{0}^{\prime} \rightarrow k_{0}}$ by this schedule. On the other hand, the minimum number of beam collisions given by Theorem 1 is

$$
\begin{aligned}
n_{c}^{\star} & =\sum_{e_{k, k^{\prime}} \in E_{o}}\left(\bar{d}_{k \rightarrow k^{\prime}}+\bar{d}_{k^{\prime} \rightarrow k}-N\right) \\
& =\sum_{e_{k, k^{\prime}} \in E_{o} \backslash\left\{e_{k_{0}, k_{0}^{\prime}}\right\}}\left(\bar{d}_{k \rightarrow k^{\prime}}+\bar{d}_{k^{\prime} \rightarrow k}-N\right) \\
& +\left(\bar{d}_{k_{0} \rightarrow k_{0}^{\prime}}+\bar{d}_{k_{0}^{\prime} \rightarrow k_{0}}-N\right)=n_{r e s}^{\star}+\bar{d}_{k_{0}^{\prime} \rightarrow k_{0}},
\end{aligned}
$$

which is obviously reached by the schedule.

The similar proof can be adopted if $k_{0}^{\prime}=0$.

(iii) $G_{o}$ is acyclic, all $\bar{d}_{k \rightarrow k^{\prime}}<N$.

Since the recursion condition (10) is satisfied, we can acquire the schedule of the first time slot with no beam collision, according to Lemma 3. Then, according to our 
inductive assumption on $N$, the rest $(K, N-1)$ problem can be solved with the minimum number of beam collisions

$$
n_{r e s}^{\star}=\sum_{e_{k, k^{\prime}} \in E_{o}^{\prime}}\left(\bar{d}_{k \rightarrow k^{\prime}}+\bar{d}_{k^{\prime} \rightarrow k}-(N-1)\right) \text {. }
$$

Next according to Lemma 3 again, we have

$$
\begin{aligned}
n_{c}^{\star} & =\sum_{e_{k, k^{\prime}} \in E_{o}}\left(\bar{d}_{k \rightarrow k^{\prime}}+\bar{d}_{k^{\prime} \rightarrow k}-N\right) \\
& =\sum_{e_{k, k^{\prime}} \in E_{o}^{\prime}}\left(\bar{d}_{k \rightarrow k^{\prime}}^{\prime}+\bar{d}_{k^{\prime} \rightarrow k}^{\prime}-(N-1)\right)=n_{r e s}^{\star} .
\end{aligned}
$$

Therefore, $n_{c}^{\star}$ is reached.

\section{REFERENCES}

[1] T. S. Rappaport, et al.,"Millimeter wave mobile communications for 5G cellular: It will work!” IEEE Access, vol. 1, pp. 335-349, May 2013.

[2] R. W. Heath, et al., "An overview of signal processing techniques for millimeter wave MIMO system," IEEE J. Sel. Topics Signal Process., vol. 10, no. 3, pp. 436-453, Apr. 2016.

[3] M. R. Akdeniz, et al., "Millimeter wave channel modeling and cellular capacity evaluation," IEEE J. Sel. Areas Commun., vol. 32, no. 6, pp. 1164-1179, Jun. 2014.

[4] M. Xiao, et al., "Millimeter wave communications for future mobile networks," IEEE J. Sel. Areas Commun., vol. 35, no. 9, pp. 1909-1935, Sep. 2017.

[5] S. Kutty and D. Sen, "Beamforming for millimeter wave communications: An inclusive survey," IEEE Commun. Surveys Tuts., vol. 18, no. 2, pp. 949-973, 2016.

[6] M. Giordani, M. Mezzavilla, and M. Zorzi, "Initial access in 5G mmWave cellular networks," IEEE Commun. Mag., vol. 54, no. 11, pp. 40-47, Nov. 2016.

[7] V. Raghavan, et al., "Beamforming tradeoffs for initial UE discovery in millimeter-wave MIMO systems," IEEE J. Sel. Topics Signal Process., vol. 10, no. 3, pp. 543-559, Apr. 2016.

[8] Q. Xue, X. Fang, M. Xiao, S. Mumtaz, and J. Rodriguez, "Beam management for millimeter-wave beamspace MU-MIMO systems," IEEE Trans. Commun., vol. 67, no. 1, pp. 205-217, Jan. 2019.

[9] S. Singh, R. Mudumbai, and U. Madhow, "Interference analysis for highly directional 60-GHz mesh networks: The case for rethinking medium access control," IEEE/ACM Trans. Networking, vol. 19, no. 5 , pp. 1513-1527, Oct. 2011.

[10] T. Bai and R. W. Heath, "Coverage and rate analysis for millimeterwave cellular networks," IEEE Trans. Wireless Commun., vol. 14, no. 2, pp. 1100-1114, Feb. 2015.

[11] M. D. Renzo, "Stochastic geometry modeling and analysis of multitier millimeter wave cellular networks," IEEE Trans. Wireless Commun. vol. 14, no. 9, pp. 5038-5057, Sep. 2015.

[12] M. Rebato, et al., "Understanding noise and interference regimes in 5G millimeter-wave cellular networks," in Proc. European Wireless 2016 (Oulu, Finland), May 18-20, 2016, pp. 84-88.

[13] G. Y. Li, et al., "Multi-cell coordinated scheduling and MIMO in LTE," IEEE Commun. Surveys Tuts., vol. 16, no. 2, pp. 761-775, 2014.

[14] V. R. Cadambe and S. A. Jafar, "Interference alignment and degrees of freedom of the K-user interference channel," IEEE Trans. Inf. Theory, vol. 54, no. 8, pp. 3425-3441, Aug. 2008.

[15] C. Wang, et al., "Low complexity interference alignment for mmWave MIMO channels in three-cell mobile network," IEEE J. Sel. Areas Commun., vol. 35, no. 7, pp. 1513-1523, Jul. 2017.

[16] M. Kim and Y. H. Lee, "MSE-Based hybrid RF/baseband processing for millimeter-wave communication systems in MIMO interference channels," IEEE Trans. Veh. Technol., vol. 64, no. 6, pp. 2714-2720, Jun. 2015.

[17] H. Shokri-Ghadikolaei, et al., "Spectrum sharing in mmWave cellular networks via cell association, coordination, and beamforming," IEEE $J$. Sel. Areas Commun., vol. 34, no. 11, pp. 2902-2917, Nov. 2016.

[18] W. Feng, et al., "When mmWave communications meet network densification: A scalable interference coordination perspective," IEEE J. Sel. Areas Commun., vol. 35, no. 7, pp. 1459-1471, Jul. 2017.

[19] J. Qiao, X. Shen, J. W. Mark, and Y. He, "MAC-layer concurrent beamforming protocol for indoor millimeter-wave networks," IEEE Trans. Veh. Technol., vol. 64, no. 1, pp. 327-338, Jan. 2015.
[20] G. Dartmann, X. Gong, and G. Ascheid, "Application of graph theory to the multicell beam scheduling problem," IEEE Trans. Veh. Technol., vol. 62 , no. 4, pp. 1435-1449, May 2013.

[21] P. Zhou, et al., "Deep learning-based beam management and interference coordination in dense mmWave networks," IEEE Trans. Veh. Technol.,vol. 68, no. 1, pp. 592-603, Jan. 2019.

[22] E. Castaneda, A. Silva, A. Gameiro, and M. Kountouris, "An overview on resource allocation techniques for multi-user MIMO systems," IEEE Commun. Surveys Tuts., vol. 19, no. 1, pp. 239-284, 2017.

[23] R. Kim, Y. Kim, and H. Lim, "Inter-BS interference-aware transmission coordination for millimeter wave networks," IEEE Wireless Commun. Lett., vol. 6, no. 3, pp. 350-353, Jun. 2017.

[24] K. Aldubaikhy and X. Shen, "Simultaneous DL transmission in mmWave ultra dense networks: Inter-BSS interference prospective," in Proc. 2018 ICC Workshops (Kansas City, MO, USA), May 20-24, 2018, pp. 1-6.

[25] 3GPP, TS 36.423, "X2 application protocol (X2AP)", V15.3.0, Sep. 2018.

[26] R. Diestel, Graph Theory. New York: Springer-Verlag, 2005.

[27] 3GPP, TR 38.900, "Study on channel model for frequency spectrum above $6 \mathrm{GHz}$ (Release 15),” V15.0.0, Jun. 2018. 